\title{
ANÁLSIS CLÍNICO Y ECONÓMICO DE LA HISTERECTOMÍA ABDOMINAL VERSUS LA HISTERECTOMÍA VAGINAL EN EL HOSPTALCÚNICO DE LA UNIVERSIDAD DE CHILE. REVISIÓN DE 2.338 CASOS
}

\author{
Hugo Salinas P.1, Jorge Pastén M. ${ }^{1}$, Benjamín Naranjo D. ${ }^{1}$, Sergio Carmona ${ }^{1}{ }^{2}$, Beatriz \\ Retamales M. ${ }^{a}$, Gonzalo Díaz V. ${ }^{a}$, Lenka Franulic C. ${ }^{a}$ \\ ${ }^{1}$ Departamento de Obstetricia y Ginecología, Hospital Clínico de la Universidad de Chile. ${ }^{2}$ Departamento de Obstetricia \\ y Ginecología, Hospital Clínico San Borja Arriarán.
}

anternos, Escuela de Medicina, Universidad de Chile.

\section{RESUMEN}

Antecedentes: La histerectomía es una intervención quirúrgica frecuente y de alto costo. Después de la operación cesárea, es el segundo procedimiento quirúrgico más frecuente en ginecología. Objetivos: Evaluar la relación costo/efectividad de la histerectomía vaginal versus la histerectomía abdominal. Método: Estudio retrospectivo observacional, en 2.338 pacientes sometidas a histerectomía por patología benigna en el Servicio de Ginecología del Hospital Clínico de la Universidad de Chile, en el período comprendido entre enero de 1997 a diciembre de 2005. Se analizaron variables clínicas y de costo entre las dos vías de abordaje. Resultados: La edad de las pacientes fluctuó entre 27 y 86 años. De las 2.338 histerectomías, la vía vaginal correspondió al 36,9\% y la abdominal en el 63,1\%; con una tasa de complicaciones de 29,8/ 100 mujeres operadas vaginalmente y de 42,8/100 mujeres operadas abdominalmente. Conclusión: Este estudio sugiere que el abordaje vaginal se asoció a tiempos operatorios más cortos, menor número de complicaciones y a costos más bajos que la vía abdominal.

\section{PALABRAS CLAVE: Histerectomía vaginal, histerectomía abdominal, costo-efectividad histerectomías}

\section{SUMMARY}

Background: After cesarean delivery, hysterectomy it is the second most frequently performed major surgical procedure. Objective: To study de relation cost/effectivity between vaginal and abdominal hysterectomy. Method: Retrospective and observational study in 2338 hysterectomy, performed between January 1997 and December 2005. Clinical and cost variables were analysed between both surgical routes. Results: The study included 2338 patients aged 27 to 86 years in whom hysterectomy was performed for benign gynaecologic disorders. Of 2338 hysterectomies, $36.9 \%$ were performed vaginally and an abdominal approach was required in $63.1 \%$. The overall complication rate was 29.8 per 100 women for vaginal hysterectomy and 42.8 per 100 women for abdominal hysterectomy. Conclusion: This study suggests that vaginal route presents some advantages in comparison to abdominal hysterectomy that include less expensive, fewer complications, shorter operating times and shorter stays.

KEYWORDS: Vaginal hysterectomy, abdominal hysterectomy, cost-effectiveness hysterectomy 


\section{INTRODUCCIÓN}

La histerectomía es una de las cirugías más frecuentes dentro de la ginecología por lo que posee importantes implicancias en el ámbito médico y económico $(1,2,3)$. Tradicionalmente la resección uterina se ha realizado por abordaje abdominal o vaginal. Cuando no existen contraindicaciones es recomendable hacerlo por abordaje vaginal, debido fundamentalmente a su menor morbilidad, menor período de convalecencia y disminución de la estadía hospitalaria (4).

En los últimos años debido a la aparición de nuevas técnicas, se hace necesario medir los efectos médicos y económicos de las distintas alternativas quirúrgicas existentes para la resección uterina (5). Es razonable pensar que cada una de estas técnicas presenta ventajas y desventajas respecto de la otra, dependiendo del motivo por el cual está indicada. Por otra parte, resulta difícil establecer una comparación directa entre las técnicas quirúrgicas, debido a la gran diversidad de criterios existentes por parte de los ginecólogos para optar por cada una de ellas (6).

El objetivo de este estudio es evaluar y comparar estas dos alternativas quirúrgicas existentes para la histerectomía realizadas en nuestro hospital, considerando variables epidemiológicas, médicas, quirúrgicas y postquirúrgicas, además de contrastar el costo de producción de cada intervención, con los valores reales de las histerectomías realizadas en el período estudiado.

\section{MATERIAL Y MÉTODO}

Se realizó un estudio retrospectivo observacional de las pacientes sometidas a histerectomía en el Hospital Clínico de la Universidad de Chile. Se compara la histerectomía vaginal (HV) con la abdominal (HA), indicadas por patología ginecológica benigna, durante el período comprendido entre enero de 1997 hasta diciembre de 2005. Cada paciente fue ingresada a una base de datos File Maker 8.0 previo a la cirugía, creando campos con distintas variables epidemiológicas, cuantitativas y cualitativas. Posteriormente se analizan las distintas variables relacionadas al procedimiento quirúrgico, como tiempo operatorio y complicaciones durante el procedimiento; específicamente pérdidas sanguíneas, lesión vesical, lesión de vía urinaria, lesión intestinal y tasa de conversión. Luego se analiza las complicaciones postoperatorias, como fiebre, tromboembolismo, infección urinaria, neumonía, hematomas pélvicos, shock, sepsis, íleo, dolor postoperatorio (medido a través de una escala métrica de rango 0-10 de acuerdo a la intensidad del dolor referido por la paciente), necesidad de transfusión sanguínea, infección de herida operatoria, ingreso a Unidad de Cuidados Intensivos, necesidad de uso de analgesia endovenosa mayor de 24 horas, uso de antibióticos endovenoso, proporción de pacientes que requirió exámenes postoperatorios, necesidad de reintervención y necesidad de interconsultas a otras especialidades. Finalmente se realizó un análisis comparativo de los costos de producción de las diferentes modalidades quirúrgicas con los costos reales, incluyendo los días de hospitalización, los exámenes de laboratorio, los procedimientos, los medicamentos e insumos requeridos durante la hospitalización, la intervención quirúrgica propiamente tal y en general todos los procedimientos relacionados que significaron algún costo para el hospital y/o los pacientes. Las distintas variables fueron analizadas mediante el software estadístico STATA 8.0 a través de $t$ test de comparación de dos medias y test de Fisher para proporción de medias. Las diferencias fueron consideradas estadísticamente significativas con un valor $p<0,05$.

\section{RESULTADOS}

Se realizaron $1.474(63,1 \%)$ histerectomías abdominales y $864(36,9 \%)$ histerectomías vaginales. En la Tabla I se muestra las distintas variables estudiadas; la realización de otros procedimientos quirúrgicos fue significativamente mayor en la HV.

Los diagnósticos asociados a la intervención quirúrgica se muestran en la Tabla II. Se destaca que la vía vaginal fue privilegiada cuando el diagnóstico preoperatorio se asoció a prolapso $(50,57 \%)$ o incontinencia urinaria $(16,34 \%)$, mientras que en la vía abdominal fue $2,03 \%$ y $1,08 \%$, respectivamente.

La comparación entre las variables edad, tiempo operatorio y costos, expresados en media, para la HV e HA se presenta en la Tabla IIla. La media de edad de las pacientes sometidas a HA fue de 47,3 años y 54,9 años para la HV, diferencia estadísticamente significativa. La media del tiempo operatorio para la HV fue de 98 minutos y para la HA de 119 minutos, diferencia estadísticamente significativa. La media de estadía hospitalaria fue 2,79 días para la HV y 3,19 días para la HA, diferencia estadísticamente significativa. El análisis de los costos muestra que el gasto en la HA es mayor y las diferencias de costos son estadísticamente significativas; el costo de producción al 
Tabla I

VARIABLES ANALIZADAS POR VÍA DE HISTERECTOMÍA. HOSPITAL CLÍNICO UNIVERSIDAD DE CHILE, 1997-2005

\begin{tabular}{|c|c|c|}
\hline Características de las pacientes & Histerectomía vaginal & Histerectomía abdominal \\
\hline $\begin{array}{l}\text { Promedio de edad } \pm \text { DS } \\
\text { rango (años) }\end{array}$ & $\begin{array}{l}54,96 \pm 12,3 \\
(31-86)\end{array}$ & $\begin{array}{l}47,27 \pm 7,23 \\
(27-83)\end{array}$ \\
\hline $\begin{array}{l}\text { Promedio tiempo operatorio } \pm \mathrm{DS} \\
\text { rango (minutos) }\end{array}$ & $\begin{array}{l}98,86 \pm 37,41 \\
(30-240,4)\end{array}$ & $\begin{array}{l}118,98 \pm 34,28 \\
(30-240,5)\end{array}$ \\
\hline Complicaciones inmediatas \% (n)* & $6,01(52)$ & $5,97(88)$ \\
\hline Conversión quirúrgica \% (n) & $6,01(52)$ & No corresponde \\
\hline $\begin{array}{l}\text { Complicaciones post cirugía } \%(n)^{* *} \\
\text { - dolor moderado a severo }(\geq 4) \\
\text { - transfusión } \\
\text { - infección herida } \\
\text { - complicación médica }{ }^{* * *} \\
\text { - reoperación }\end{array}$ & $\begin{array}{l}29,86(258) \\
24,65(213) \\
1,38(12) \\
0,23(2) \\
3,00(26) \\
0,57(5)\end{array}$ & $\begin{array}{l}42,87(632) \\
33,98(501) \\
0,81(12) \\
2,03(30) \\
5,02 \%(74) \\
1,01 \%(15)\end{array}$ \\
\hline Estadía Unidad Cuidados Intensivos \% (n) & $0,57(5)$ & $1,22(18)$ \\
\hline $\begin{array}{l}\text { Estadía hospitalaria días } \pm \text { DS } \\
\text { rango (días) }\end{array}$ & $\begin{array}{l}2,79 \pm 1,72 \\
(2-20)\end{array}$ & $\begin{array}{l}3,19 \pm 2,08 \\
(3-21)\end{array}$ \\
\hline Exámenes postoperatorios \% (n) & $10,99(95)$ & $17,97(265)$ \\
\hline Tratamiento antibiótico endovenoso \% (n) & $6,01(52)$ & $14,99(221)$ \\
\hline Analgesia endovenosa $>24$ horas $\%(n)$ & $7,98(69)$ & $13,97(206)$ \\
\hline $\begin{array}{l}\text { Interconsulta especialidad \% ( } \mathrm{n}) \\
\text { - en el preoperatorio } \\
\text { - en el postoperatorio }\end{array}$ & $\begin{array}{l}32,06(277) \\
4,05(35)\end{array}$ & $\begin{array}{l}21,03(310) \\
5,97(88)\end{array}$ \\
\hline Otros procedimientos $\%(n)^{* \star \star *}$ & $29,05(251)$ & $16,01(236)$ \\
\hline $\begin{array}{l}\text { Costos reales en pesos chilenos } \pm \text { DS } \\
\text { rango (pesos chilenos) }\end{array}$ & $\begin{array}{l}899.169 \pm 54.884 \\
(844.285-954.052)\end{array}$ & $\begin{array}{l}1.061 .879 \pm 105.847 \\
(956.033-1.167 .726)\end{array}$ \\
\hline Costos teóricos de producción en pesos chilenos ${ }^{\#}$ & 760.957 & 928.446 \\
\hline
\end{tabular}

DS: Desviación estándar

* Pérdidas sanguíneas importantes, lesión vesical, lesión vía urinaria, lesión intestinal.

** Dolor moderado a severo, anemia que requiere transfusión, infección herida, complicación médica, reintervención.

${ }^{* \star \star}$ Fiebre, tromboembolismo, infección urinaria, neumonía, hematomas pélvicos, shock, sepsis, íleo.

${ }^{* \star \star}$ Cinta para incontinencia de orina, abdominoplastía, reparación de prolapso anterior, reparación de prolapso posterior, fijación de cúpula vaginal, apendicectomía.

\# Días cama ocupados, exámenes, intervención quirúrgica, procedimientos, medicamentos e insumos.

compararlo con el costo real de cada una de las alternativas, es significativamente menor en la HV.

El análisis comparativo de morbilidad se presenta en la Tabla Illb. No se encontraron diferencias estadísticamente significativas en relación a las complicaciones inmediatas derivadas del acto quirúrgico (pérdida sanguínea importante, lesión vesical, lesión vía urinaria, lesión intestinal). Estas fueron de $6,01 \%$ para HV y $5,97 \%$ para HA. El porcentaje de conversión de la HV fue de 6,01\%. Las complicaciones post operatorias se presentaron de manera significativamente mayor en la HA. El dolor postoperatorio moderado a severo fue significativamente mayor en la ruta abdominal. La necesidad de analgesia endovenosa por un tiempo mayor de 24 horas es concordante con lo anterior y fue significativamente mayor en la HA. Existen diferencias significativas respecto del uso 
Tabla II

DIAGNÓSTICOS PREOPERATORIOS EN PACIENTES SOMETIDAS A HISTERECTOMÍA POR PATOLOGÍA BENIGNA SEGÚN VÍA DE ACCESO. HOSPITAL CLÍNICO UNIVERSIDAD DE CHILE, 1997-2005

\begin{tabular}{lcc}
\hline Diagnósticos preoperatorios * & Histerectomía vaginal & Histerectomía abdominal \\
& $n(\%)$ & $993(67,36)$ \\
\hline Mioma uterino & $399(46,18)$ & $216(14,65)$ \\
Lesión anexial & $15(1,73)$ & $93(6,30)$ \\
Adenomiosis & $74(8,56)$ & $53(3,59)$ \\
Anemia secundaria & $15(1,73)$ & $47(3,18)$ \\
Metrorragia/hipermenorrea & $25(2,89)$ & $40(2,71)$ \\
Hiperplasia/engrosamiento endometrial & $43(4,97)$ & $30(2,03)$ \\
Prolapso & $437(50,57)$ & $27(1,83)$ \\
Endometriosis & $3(0,34)$ & $26(1,76)$ \\
Enfermedad inflamatoria pélvica & $2(0,23)$ & $22(1,49)$ \\
Absceso tuboovárico & $0(0,00)$ & $18(1,22)$ \\
Algia pélvica & $9(1,04)$ & $16(1,08)$ \\
Incontinencia de orina & $142(16,34)$ & $13(0,88)$ \\
Pólipos & $17(1,96)$ & $10(0,67)$ \\
Retroversión uterina & $4(0,46)$ & $6(0,40)$ \\
Hematometra & $0(0,00)$ & $4(0,27)$ \\
Desgarro perineal & $8(0,92)$ & $3(0,20)$ \\
Elongación/desgarro cervical & $6(0,69)$ & $1(0,06)$ \\
Quiste vaginal & $1(0,11)$ & $1(0,06)$ \\
Útero doble & $0(0,00)$ & $0(0,00)$ \\
Hemoperitoneo & $2(0,23)$ & $0(0,00)$ \\
Endometritis crónica & $2(0,23)$ & $0(0,00)$ \\
Dismenorrea & $1(0,11)$ &
\end{tabular}

*Algunos pacientes tienen múltiples diagnósticos. En algunos casos no necesariamente los diagnósticos son la indicación de la histerectomía.

Tabla Illa

ANÁLISIS DE LAS VARIABLES DE ACUERDO A LA VÍA DE HISTERECTOMÍA. HOSPITAL CLÍNICO UNIVERSIDAD DE CHILE, 1997-2005

\begin{tabular}{lllll}
\hline Variables & Media & $D S$ & IC 95\% & Valor $p$ \\
\hline Edad (años) & & & & \\
HV & 54,9 & 12,3 & $54,13-55,78$ & $<0,0001$ \\
HA & 47,2 & 7,2 & $46,90-47,63$ & \\
Tiempo operatorio (min) & & & & \\
HV & 98,8 & 37,4 & $96,36-101,35$ & $<0,0001$ \\
HA & 118,9 & 34,2 & $117,22-120,73$ & \\
Estadía hospitalaria (días) & & & & \\
HV & 2,7 & 1,7 & $2,67-2,90$ & $<0,0001$ \\
HA & 3,1 & 2,0 & $3,08-3,29$ & \\
Costos reales (\$) & & & & \\
HV & 889.169 & 54.884 & $1.056 .471-1.067 .287$ & \\
HA & 1.061 .879 & 105.847 & & $<0,0001$ \\
Costos (\$) reales/teóricos & & & $760.957-760.957$ & $<0,0001$ \\
HVt/HV & 760.957 & 0 & $885.504-892.833$ & $<0,0001$ \\
HVt/HA & 889.169 & 54.884 & $928.446-928.446$ & $<0,0001$ \\
HAt/HV & 928.446 & 0 & $1.056 .471-1.067 .287$ & $<0,0001$ \\
HAt/HA & 1.061 .879 & 105.847 & & \\
\hline
\end{tabular}

\$: Pesos chilenos. t: Teórico. HV: Histerectomía vaginal. HA: Histerectomía abdominal. DS: Desvío estándar. 
Tabla IIlb

ANÁLISIS DE LAS VARIABLES DE ACUERDO A LA Vía DE HISTERECTOMÍA. HOSPITAL CLínICO UNIVERSIDAD DE CHILE, 1997-2005

\begin{tabular}{|c|c|c|c|}
\hline Variables & $n$ & $\%$ & Valor $p^{*}$ \\
\hline \multicolumn{4}{|c|}{ Complicaciones inmediatas } \\
\hline HV & 52 & 6,01 & NS \\
\hline $\mathrm{HA}$ & 88 & 5,97 & \\
\hline \multicolumn{4}{|c|}{ Conversión quirúrgica } \\
\hline HV & 52 & 6,01 & NA \\
\hline \multicolumn{4}{|c|}{ Complicaciones postoperatorias } \\
\hline $\mathrm{HV}$ & 258 & 29,86 & $<0,001$ \\
\hline HA & 632 & 42,87 & \\
\hline \multicolumn{4}{|l|}{ Dolor $(\geq 4)$} \\
\hline HV & 213 & 24,65 & $<0,001$ \\
\hline HA & 632 & 33,98 & \\
\hline \multicolumn{4}{|c|}{ Transfusión } \\
\hline HV & 12 & 1,38 & 0,026 \\
\hline $\mathrm{HA}$ & 12 & 0,81 & \\
\hline \multicolumn{4}{|c|}{ Infección herida } \\
\hline HV & 2 & 0,23 & $<0,001$ \\
\hline HA & 30 & 2,03 & \\
\hline \multicolumn{4}{|c|}{ Complicaciones médicas } \\
\hline $\mathrm{HV}$ & 26 & 3,00 & 0,026 \\
\hline HA & 74 & 5,02 & \\
\hline \multicolumn{4}{|c|}{ Analgesia ev } \\
\hline HV & 69 & 7,98 & $<0,001$ \\
\hline $\mathrm{HA}$ & 206 & 13,97 & \\
\hline \multicolumn{4}{|c|}{ Reoperación } \\
\hline HV & 5 & 0,57 & NS \\
\hline $\mathrm{HA}$ & 15 & 1,01 & \\
\hline \multicolumn{4}{|c|}{ Estadía en UCI } \\
\hline HV & 5 & 0,57 & NS \\
\hline $\mathrm{HA}$ & 18 & 1,22 & \\
\hline \multicolumn{4}{|c|}{ Exámenes postoperatorios } \\
\hline HV & 95 & 10,99 & $<0,001$ \\
\hline $\mathrm{HA}$ & 265 & 17,97 & \\
\hline \multicolumn{4}{|c|}{ Tratamiento antibiótico } \\
\hline $\mathrm{HV}$ & 52 & 6,01 & $<0,001$ \\
\hline $\mathrm{HA}$ & 221 & 14,99 & \\
\hline \multicolumn{4}{|c|}{ IC especialidad } \\
\hline HV & 35 & 4,05 & NS \\
\hline HA & 88 & 5,97 & \\
\hline \multicolumn{4}{|c|}{ Otros procedimientos } \\
\hline $\mathrm{HV}$ & 251 & 29,05 & $<0,001$ \\
\hline $\mathrm{HA}$ & 236 & 16,01 & \\
\hline
\end{tabular}

${ }^{*}$ Test de Fisher.

HV: Histerectomía vaginal. HA: Histerectomía abdominal.

NS: No significativo.

NA: No aplicable. ev: Endovenoso.

UCI: Unidad de cuidados intensivos. IC: interconsulta. 


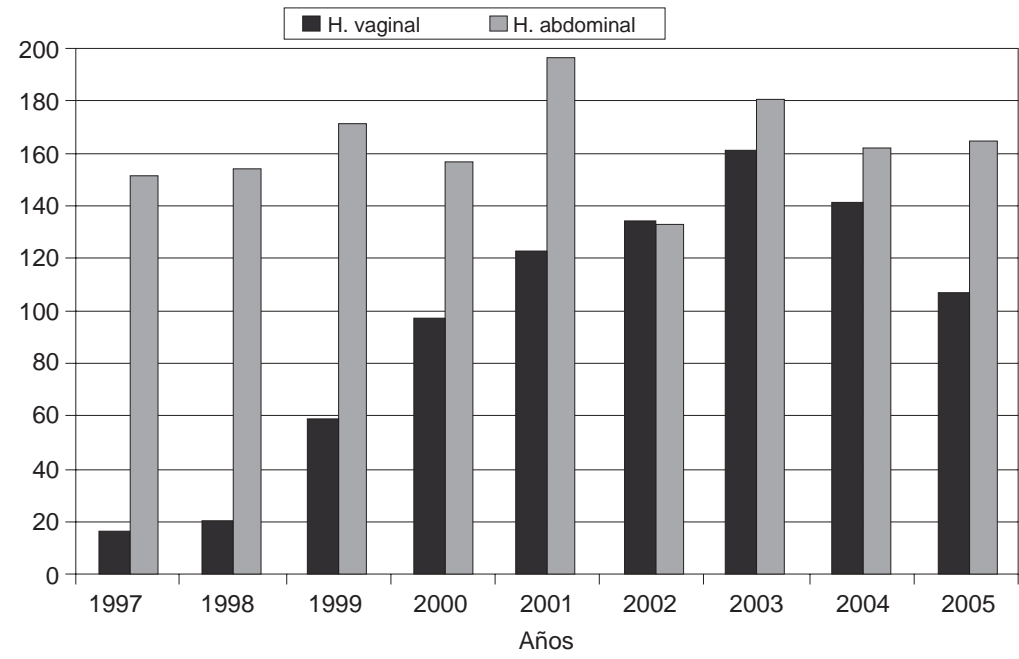

Figura 1. Número de histerectomías por vía de acceso, Hospital Clínico de la Universidad de Chile, 1997-2005. de tratamiento antibiótico endovenoso, siendo más requerido en las pacientes que se realizó HA. La infección de la zona operatoria (infección de sitio operatorio y de cúpula vaginal) fue significativamente mayor en la HA. El $15,44 \%$ de las histerectomías, debido a su evolución, requirió exámenes postoperatorios, siendo significativamente mayor en la HA.

La evolución de las diferentes alternativas de histerectomía en el período estudiado se presenta en la Figura 1.

\section{DISCUSIÓN}

Este estudio estuvo orientado al análisis de distintas variables de pacientes sometidas a histerectomía. Los datos obtenidos sugieren que la HV, cuando no está contraindicada, es la vía de acceso recomendable para realizar una histerectomía, por sobre la HA, debido fundamentalmente a su menor morbilidad asociada, menor estadía hospitalaria y menor costo de producción y de gastos. Esto último propone a la HV en relación a la HA, como la ruta más acertada cuando el abordaje por esta vía es el apropiado y no presente contraindicaciones. Al optar por la vía vaginal es importante considerar diversos factores como el tamaño uterino, la patología con compromiso extrauterino, la severidad de la patología y, lo más importante, la existencia de preparación y experiencia de parte de los especialistas para realizar la cirugía (7).

El inicio de la HV en nuestro hospital se produce a comienzos del año 1997, existiendo un constante aumento con el transcurso de los años. Esto podría ser explicado por la creciente formación y capacitación de los ginecólogos para utilizar esta vía a partir de esa fecha, tras el retorno de especialistas de nuestro hospital preparados en el extranjero. Otro aspecto importante a tener presente es que a partir de 2001 , se ha mantenido relativamente estable el porcentaje de las vías de acceso, lo que podría estar revelando una conducta estandarizada y homogénea por parte de los ginecólogos de nuestro departamento.

La HA es, naturalmente, la cirugía más agresiva para la patología uterina benigna. Del mismo modo, cualquiera sea la técnica que se utilice, se requiere de una gran experiencia por parte del ginecólogo que la realiza para obtener los mejores resultados (7).

Como conclusión, los resultados del estudio proponen que la $\mathrm{HV}$ en relación a la $\mathrm{HA}$ es la práctica de elección cuando esta es posible de realizar, debido a que se asocia a un menor número de complicaciones, costos más bajos, menor estadía hospitalaria y menor morbilidad. No obstante, el tipo de histerectomía a realizar dependerá de la decisión tomada en conjunto por la paciente y su médico tratante, de acuerdo al costobeneficio que esto implique en cada caso.

En nuestro centro no se ha realizado una revisión del seguimiento de las pacientes histerectomizadas, por lo que no logramos obtener datos en relación al tiempo requerido para el retorno a una vida normal y la calidad de vida posterior. Sin embargo, existen estudios previos de costo-efectividad que no demuestran mayores diferencias en la calidad de vida desde las primeras semanas de alta hasta meses posteriores a la cirugía $(7,8,9)$. 


\section{BIBLIOGRAFÍA}

1. Vessey MP, Villard-Mackintosh L, McPherson K, Coulter A, Yeates D. The epidemiology of hysterectomy: findings in a large cohort study. $\mathrm{Br} \mathrm{J}$ Obstet Gynaecol 1992;99:402-7.

2. Lepine LA, Hillis SD, Marchbanks PA, Koonin LM, Morrow B, Kieke BA, et al. Hysterectomy surveillance-United States 1980-1993. MMWR Surveill Summ 1997;46:1-15.

3. Graves EJ, Kozak LJ. National Hospital Discharge Survey: Annual Summary, 1996. Hyattsville, MD: National Center of Health Statistics; January 1999: Series 13, No. 140.

4. Clinch J. Length of hospital stay after vaginal hysterectomy. Br J Obstet Gynaecol 1994;10(3):253-4.

5. Garry R, Fountain J, Mason S, Napp V, Bridgman S, Hawe J, Clayton R, Abott J, et al. The eVALuate study: two parallel randomized trials, one comparing laparoscopic with abdominal hysterectomy, the other comparing laparoscopic with vaginal hysterectomy. BMJ 2004;328(7432):129-33.

6. Garry R, Fountain J, Brown J, Manca A, Mason S, Sculpher $M$, et al. Evaluate hysterectomy trial: a multicentre randomized trial comparing abdominal, vaginal and laparoscopic methods of hysterectomy. Health Technol Assess 2004;8(26):1-154.

7. Johnson N, Barlow D, Lethaby A, Tavender E, Curr L, Garry R. Methods of hysterectomy: systematic review and meta-analysis of randomized controlled trial. BM 2005;330:1478-doi:10.1136/bmj.330.7506.1478

8. Dorsey J, Holtz P, Griffiths R, McGrath M, Steinberg E. Costs and Charges associated with three alternative techniques of hysterectomy. $\mathrm{N}$ Engl $\mathrm{J}$ Med 1996:335:476-82.

9. Gupta S, Manyonda I. Hysterectomy for benign gynaecological disease. Current Cbstet Gynaecol 2006, in press. doi:10.1016/j.curobgyn.2006.04.004. 